\title{
Physical activity energy expenditure vs cardiorespiratory fitness level in impaired glucose metabolism
}

\author{
Lærke P. Lidegaard $^{1}$ • Anne-Louise S. Hansen ${ }^{2}$ Nanna B. Johansen ${ }^{1,3}$. \\ Daniel R. Witte ${ }^{2,3}$ - Søren Brage ${ }^{4}$. Torsten Lauritzen ${ }^{2}$ - Marit E. Jørgensen ${ }^{1,5}$. \\ Dirk L. Christensen ${ }^{1,4,6} \cdot$ Kristine Færch ${ }^{1}$
}

Received: 24 June 2015 / Accepted: 5 August 2015 /Published online: 5 September 2015

(C) Springer-Verlag Berlin Heidelberg 2015

\begin{abstract}
Aim/hypothesis Little is known about the relative roles of physical activity energy expenditure (PAEE) and cardiorespiratory fitness (CRF) as determinants of glucose regulation. The aim of this study was to examine the associations of PAEE and CRF with markers of glucose metabolism, and to test the hypothesis that CRF modifies the association between PAEE and glucose metabolism.

Methods We analysed cross-sectional data from 755 adults from the Danish ADDITION-PRO study. On the basis of OGTT results, participants without known diabetes were classified as having normal glucose tolerance, isolated impaired fasting glycaemia (i-IFG), isolated impaired glucose tolerance (i-IGT), combined IFG + IGT or screen-detected diabetes mellitus. Markers of insulin sensitivity and beta cell function were determined. PAEE was measured using a combined heart rate and movement sensor. CRF (maximal oxygen uptake)
\end{abstract}

Electronic supplementary material The online version of this article (doi:10.1007/s00125-015-3738-x) contains peer-reviewed but unedited supplementary material, which is available to authorised users.

Lærke P. Lidegaard

lpil@steno.dk

1 Steno Diabetes Center A/S, Niels Steensens Vej 6, DK-2820 Gentofte, Denmark

2 Department of Public Health - Institute of General Medical Practice, Aarhus University, Aarhus, Denmark

3 Danish Diabetes Academy, Odense, Denmark

4 MRC Epidemiology Unit, University of Cambridge, Cambridge, UK

5 National Institute of Public Health, University of Southern Denmark, Odense, Denmark

6 Section of Global Health, University of Copenhagen, Copenhagen, Denmark

was estimated using a submaximal 8 min step test. The associations were examined by linear regression analysis. Results were adjusted for relevant confounders.

Results PAEE and CRF were reduced in individuals with iIGT, combined IFG + IGT and screen-detected diabetes mellitus, but were not significantly different in individuals with i-IFG compared with those with normal glucose tolerance. When adjusting CRF for PAEE and vice versa, PAEE and $\mathrm{CRF}$ were both associated with lower fasting and $2 \mathrm{~h}$ insulin and higher peripheral insulin sensitivity. CRF was additionally associated with lower fasting and $2 \mathrm{~h}$ glucose and higher insulin sensitivity and beta cell function. There was no interaction between CRF and PAEE for any markers of glucose metabolism. Conclusions/interpretation Only CRF, not PAEE, appears to be independently associated with plasma glucose levels and beta cell function, suggesting that CRF may be particularly important for glycaemic control.

Keywords Beta cell function - Cardiorespiratory fitness . Energy expenditure $\cdot$ Insulin sensitivity $\cdot$ Maximum oxygen uptake $\cdot$ Physical activity $\cdot$ Prediabetes $\cdot$ Type 2 diabetes . $\dot{V} \mathrm{O}_{2 \max }$

\begin{tabular}{|c|c|}
\hline Abbreviations & \\
\hline BIGTT-AIR & $\begin{array}{l}\text { Surrogate measure of acute insulin } \\
\text { response derived from OGTT }\end{array}$ \\
\hline CRF & Cardiorespiratory fitness \\
\hline DI & Disposition index \\
\hline HOMA-IS & HOMA of insulin sensitivity \\
\hline i-IFG & Isolated impaired fasting glycaemia \\
\hline i-IGT & Isolated impaired glucose tolerance \\
\hline IFG/IGT IFG + IGT & $\begin{array}{l}\text { Combined impaired fasting glycaemia } \\
\text { and impaired glucose tolerance }\end{array}$ \\
\hline $\mathrm{ISI}_{0,120} \mathrm{ISI}_{(0}$ & Insulin sensitivity index \\
\hline
\end{tabular}

Abbreviations

$\mathrm{ISI}_{0,120} \mathrm{ISI}_{(0,120)}$ 

MET
NGT
PAEE
SDM

individual predisposition. It is estimated that $10-50 \%$ of the variance in fitness is inherited [13]. A person can therefore have a high level of CRF, expressed as maximal oxygen consumption, without necessarily engaging in high PAEE. A high level of physical activity, especially vigorous activity, improves and generally leads to a high level of CRF. However, physical activity may not increase $\mathrm{CRF}$, if the activity is mainly of low intensity [13]. Accordingly, the effect of different intensities and types of physical activity may have different effects on glucose metabolism. This has mainly been investigated in low risk populations, and the findings are equivocal [14-18]. Furthermore, studies have found that CRF changes the direction and/or strength of the association between PAEE and cardiometabolic risk factors [19, 20], suggesting that the beneficial effect of PAEE is dependent on the intensity of the activities contributing to PAEE.

As more diabetes screening programmes are deployed, high-risk populations are increasingly identified. However, it is still unclear whether risk reduction in these high-risk individuals should be approached by increasing the overall energy expenditure or by engaging in vigorous activities to increase CRF. We hypothesised that both PAEE and CRF are associated with increased insulin sensitivity and that the associations between CRF and the markers of glucose metabolism are stronger than those with PAEE. Furthermore, we hypothesised that the effect of PAEE is dependent on the level of CRF. Therefore, the aims of this study were threefold: (1) to examine whether individuals with NGT, different subtypes of prediabetes and SDM have different levels of PAEE and CRF; (2) to examine and compare the associations of PAEE vs CRF with markers of glucose metabolism; and (3) to examine whether CRF modifies the association between PAEE and glucose metabolism. IFG + IGT" These abnomitities sent distinct pathways to diabetes [6-8] and the prediabetes subtypes seem to respond differently to exercise training [9]. The different prediabetes subgroups may also have different levels of physical activity energy expenditure (PAEE) and cardiorespiratory fitness (CRF). On this background, we hypothesised that PAEE and CRF levels are lower in individuals with i-IGT, IFG + IGT and screen-detected diabetes mellitus (SDM) than in individuals with normal glucose tolerance (NGT) or i-IFG.

Currently, it is debated whether strategies to promote beneficial health effects should focus on increasing the overall energy expenditure or should promote participation in vigorous activity to increase CRF [10]. However, little is known about the relative influence of PAEE vs CRF on glucose regulation. Although PAEE and CRF seem to represent two sides of the same coin, they differ significantly from each other at crucial points. PAEE is a complex and multidimensional behaviour [11, 12] and thus more difficult to measure, whereas CRF is a more easily measurable variable, which partly represents an integral of past physical activity and partly

\section{Methods}

Study population The ADDITION-PRO study is a longitudinal cohort study and a part of the Danish arm (ADDITION-DK study) of the ADDITION-Europe study. The ADDITION-PRO study is a follow-up study of the ADDITION-DK study, which included individuals at low to high risk of developing type 2 diabetes, recruited based on a stepwise screening procedure in 2001-2006. A total of 4,188 individuals without type 2 diabetes were invited to participate in a follow-up health examination, the ADDITION-PRO study. Of these, 2,082 ( $50 \%$ of those invited) completed the health examination, which took place from 2009 to 2011 at four centres in Denmark [21]. The ADDITION-PRO study has been described in detail elsewhere [21].

A total of 955 individuals had valid data on CRF and, of these, 896 individuals also had valid data on PAEE. Participants with known type 2 diabetes at the health examination $(n=128)$, participants who were not classified into a 
glucose tolerance group $(n=8)$ and participants fasting less than $8 \mathrm{~h}(n=5)$ prior to the health examination were excluded from the analysis. In total, 755 individuals were included. The participants in this study are thus a subpopulation of the participants in previously published studies [4, 22].

The study was approved by the ethics committee of the Central Denmark Region (reference no. 20080229) and was conducted in accordance with the Helsinki Declaration. All participants provided oral and written informed consent before participating in the study.

General information and body composition Information on age and sex was obtained from the unique Danish civil registration number. At the health assessment, participants completed a health questionnaire about general health status including smoking status (current smoker, never smoker, ex-smoker) and alcohol consumption (units per week) [21]. Height was measured without shoes to the nearest millimetre using a stadiometer (Seca, Hamburg, Germany). Participants were weighed in light clothes without shoes using a body composition analyser (Tanita, Tokyo, Japan). Weight was measured to the nearest $0.1 \mathrm{~kg}$ and clothes were estimated to weigh $0.5 \mathrm{~kg}$, which was deducted from the participants' total weight. Waist circumference was measured to the nearest millimetre, at the mid-point between the lower costal margin and the level of the anterior superior iliac crest. Waist circumference was measured twice by the same person and the mean value was used [21, 22].

PAEE To measure the physical activity of the participants, a combined accelerometer and heart rate monitor (ActiHeart, CamNTech, Cambridge, UK) was used [23]. The monitor measures accelerations (recorded as counts) and heart rate independently. The monitor was placed horizontally on the participant's chest with two standard electrocardiogram electrodes (Maxensor, Alton, UK). On the day of the health examination, an 8 min submaximal step test was performed to account for individual variations in heart rate to physical activity intensity. The participants were asked to step up and down a $20.5 \mathrm{~cm}$ step bench (Rucanor Europe, Nieuwerkerk aan den IJssel, the Netherlands) following a ramped step frequency protocol starting at 15 step cycles per min and increasing to 33 cycles per min over the $8 \mathrm{~min}$ test or less if the participant was unable to complete the whole test. Heart rate recording was continued for $2 \mathrm{~min}$ of recovery (sitting). The calibration variable for heart rate to physical activity intensity was derived as described previously [24].

After the step test, the monitor was set to long-term recording. The participants were asked to wear the monitor for 7 days and nights and to maintain their usual physical activity level. Only measures from participants with a minimum of $24 \mathrm{~h}$ of combined monitor wear time were considered valid for the present analysis [21].
Accelerometer data and heart rate measures from the combined monitor were downloaded to the manufacturer's software. From the accumulated minute-by-minute measurements, two separate time series of physical activity intensity based on accelerometer and heart rate data were combined into one using branched equation modelling [24]. The resulting intensity time series were then summarised into daily PAEE $\left(\mathrm{kJ} \mathrm{kg}^{-1}\right.$ day $^{-1}$ ) [24], whilst minimising diurnal information bias. The combination of measured physical activity by accelerometer and heart rate has been shown to give more valid results than measurement of physical activity only by accelerometer or heart rate [24]. The fractions of time spent in physical activity intensity groups expressed as multiples of the resting metabolic rate (i.e. metabolic equivalents [METs]) were derived [25]. Time spent at various MET levels represent the physical activity intensity distribution across a $24 \mathrm{~h}$ spectrum [25].

Cardiorespiratory fitness The $8 \mathrm{~min}$ step test was also used to estimate the CRF level, i.e. estimated maximum oxygen uptake of the participants. CRF was estimated by extrapolating the linear regression between observed heart rate response and oxygen cost of the step test to maximal heart rate defined by the Tanaka equation $(208-0.7 \times$ age $)[25,26]$. To estimate CRF, participants had to complete a step test of a minimum of 4 min. Participants who were physically impaired or had certain cardiovascular conditions, e.g. angina pectoris, were excluded from the step test [21].

Markers of glucose metabolism After at least $8 \mathrm{~h}$ of fasting overnight, a venous blood sample was drawn. All participants without known diabetes underwent a standard OGTT (75 g glucose dissolved in $250 \mathrm{ml}$ water). Blood samples were drawn at 30 and $120 \mathrm{~min}$ after glucose intake. Plasma glucose levels were assessed using the Hitachi 912 system (Roche Diagnostics, Mannheim, Germany) or the Vitros 5600 integrated system (Ortho Clinical Diagnostic, Illkrich Cedex, France). Serum insulin was determined by an immunoassay method (AutoDELFIA; Perkin Elmer, Waltham, MA, USA) with an inter-assay precision $\mathrm{CV}<6 \%$. $\mathrm{HbA}_{1 \mathrm{c}}$ was determined by high-performance liquid chromatography (TOSOH G7, Tokyo, Japan). On the basis of the OGTT results, participants were classified as having NGT, i-IFG, i-IGT, combined IFG + IGT or SDM according to the 2006 WHO criteria [21, 27].

Two measures of insulin sensitivity were calculated. One was derived from HOMA of insulin sensitivity (HOMA-IS=1/HOMA-IR) [21, 28]. HOMA-IS represents insulin sensitivity in the fasting state, which is mainly regulated by the liver [19]. As a proxy measure of peripheral insulin sensitivity, the insulin sensitivity index $\left(\mathrm{ISI}_{0,120}\right)$ was used. This index is based on fasting and 120 min values of plasma glucose and serum insulin [28]. As a measure of absolute beta cell function, BIGTT-AIR (a surrogate measure of acute 
insulin response derived from OGTT) was calculated based on early phase insulin release [29]. We also assessed the relative insulin response, the disposition index (DI). DI is an expression of the ability of the beta cells to compensate for decreased insulin sensitivity [21]. DI was calculated by multiplying BIGTT-AIR with ISI ${ }_{0,120}$ [4].

Statistical analyses To examine differences in levels of PAEE and CRF between glycaemic groups, an overall ANOVA was performed, and if significant, post hoc $t$ tests were used to study pairwise differences in the means or geometrical means. Differences between groups were tested with and without adjustment for age, sex and waist circumference. Both PAEE and CRF were logarithmically transformed before analysis to fulfil the assumption of normality of the residuals. The associations of PAEE and CRF with markers of glucose metabolism were explored by linear regression analysis. The following markers were log-transformed: fasting and $2 \mathrm{~h}$ serum insulin, HOMAIS, ISI ${ }_{0,120}$, BIGTT-AIR and DI. For each outcome, three different models were fitted. The first model was adjusted for age and sex, the second model included further adjustment for smoking status and alcohol consumption, and the third model was additionally adjusted for waist circumference. The three models were repeated using first PAEE and then CRF as exposure variable.

To examine whether CRF modified the association between PAEE and glucose metabolism, a multiplicative interaction term between PAEE and CRF was added to the fully adjusted model (Model 3) after dividing CRF into deciles from the 10\% smallest numbers up to the $10 \%$ largest. A likelihood ratio test was used to quantify evidence for an interaction. To assess the independent association of the two exposures and to explore whether the association with glucose metabolism was different between PAEE and CRF, a fourth model was fitted. This model included PAEE and CRF as well as all the confounders from Model 3. To enable comparison between the beta coefficients from the models with PAEE vs CRF, standardised PAEE and CRF were used in all models. All data were handled and analysed in SAS version 9.2 (SAS Institute, Cary, NC, USA). The study used a two-sided statistical significance level of 5\%.

\section{Results}

Characteristics of the study population A total of $36.3 \%$ out of 2,082 participants from the ADDITION-PRO study were included in this study $(n=775)$ of whom $44.9 \%$ were women. The mean age of the participants was 65.4 years (interquartile range $60.8,70.7)$. Excluded participants did not differ in terms of age, sex, smoking status, $\mathrm{HbA}_{1 \mathrm{c}}$ or in relation to glucose and insulin concentrations or waist circumference. The excluded participants were less likely to be employed and reported less alcohol consumption. Furthermore, the excluded participants were significantly more likely to take anti-hypertensive and lipid-lowering drugs than the included participants (electronic supplementary material [ESM] Table 1). The average wear time of the combined monitor was 6.6 days $(95 \%$ CI $6.5,6.7)$ for included and 6.2 days $(95 \%$ CI $6.1,6.3)$ for excluded individuals, which was significantly different.

Demographic, clinical and health characteristics of the study population stratified by glucose tolerance status are presented in Table 1. Eighty-one participants were identified as having diabetes at the health examination. More men than women had i-IFG and SDM. Apart from smoking status and occupational status, an overall difference was found between the groups for all of the variables listed in Table 1. A total of $140(18.6 \%)$ of the participants were taking lipid-lowering drugs and 267 (35.4\%) participants were taking antihypertensive drugs. Lower insulin levels, insulin sensitivity and beta cell function were associated with higher levels of glycaemia (Table 1).

Physical activity according to glucose tolerance status In Fig. 1, the physical activity characteristics of the study population by glucose tolerance status are shown. There was no overall difference in mean wear time of the combined monitor between the glucose tolerance groups $(p=0.983)$. The median PAEE was $12.5-22.5 \%$ lower in the i-IGT, IFG + IGT and SDM groups than the NGT group, but was not different between the i-IFG and NGT groups. After adjusting PAEE for age, sex and waist circumference, the difference between the i-IGT group and the groups with NGT and i-IFG attenuated and was no longer statistically significant; however, the lower PAEE levels in the groups with IFG + IGT and SDM remained significant (data not shown). The i-IGT and IFG + IGT and SDM groups had the lowest levels of CRF, whereas the level of CRF was similar in the i-IFG and NGT groups. Adjustment for age, sex and waist circumference did not change these findings. Over a period of $24 \mathrm{~h}$, the i-IGT, IFG + IGT and SDM groups only spent $2.6-2.7 \%$ of time in activities of moderate-tovigorous intensity ( $\geq 3$ METs). This proportion of time was significantly lower than that for both NGT and i-IFG $(p<0.01)$. After adjusting for age, sex and waist circumference, only the groups with IFG + IGT and SDM spent significantly less time in activities of moderate-to-vigorous intensity than the NGT group (Fig. 1).

Associations of PAEE vs CRF with glucose metabolism Both PAEE and CRF were found to be associated with lower fasting and $2 \mathrm{~h}$ serum insulin concentrations and higher insulin sensitivity and DI after adjustment for age, sex, smoking, alcohol consumption and waist circumference (Table 2, Model 3). Neither PAEE nor CRF was associated with BIGTT-AIR or $\mathrm{HbA}_{1 \mathrm{c}}$ upon adjustment for waist circumference. 
Table 1 Characteristics of the study population by glucose tolerance status

\begin{tabular}{|c|c|c|c|c|c|c|}
\hline Characteristic & NGT & i-IFG & i-IGT & $\mathrm{IFG}+\mathrm{IGT}$ & SDM & $p$ \\
\hline$n(\%)$ & $407(53.9 \%)$ & $146(19.3 \%)$ & $54(7.2 \%)$ & $67(8.9 \%)$ & $81(10.7 \%)$ & \\
\hline Women, $n(\%)$ & $210(51.6 \%)$ & $50(34.3 \%)$ & $28(51.9 \%)$ & $29(43.3 \%)$ & $22(27.2)$ & $<0.001$ \\
\hline Age (years) & $65.5(60.1,70.3)$ & $66.1(61.2,70.7)$ & $69.8(63.9,73.9)$ & $66.3(61.7,69.7)$ & $64.9(60.8,69.0)$ & $<0.01$ \\
\hline Employment, $n(\%)^{\mathrm{a}}$ & $225(53.1 \%)$ & $77(18.2 \%)$ & $39(9.2 \%)$ & $38(9.0 \%)$ & $45(10.6 \%)$ & 0.543 \\
\hline Smoking, $n$ (\% yes) & $64(15.7 \%)$ & $18(12.3 \%)$ & $13(24.5 \%)$ & $6(9.0 \%)$ & $16(20.3 \%)$ & 0.175 \\
\hline Alcohol (units/week) & $6.0(3.0,12.0)$ & $7.0(4.0,18.0)$ & $11.0(4.0,18.0)$ & $10.0(4.0,17.0)$ & $8.0(3.0,19.0)$ & $<0.001$ \\
\hline Waist circumference (cm) & $90.8(82.5,99.3)$ & $100.0(91.8,106.5)$ & $93.4(84.3,103.8)$ & $102.8(92.0,108.3)$ & $103.0(95.8,105.0)$ & $<0.001$ \\
\hline Fasting plasma glucose (mmol/l) & $5.6(5.3,5.8)$ & $6.4(6.2,6.6)$ & $5.7(5.6,7.1)$ & $6.5(6.3,6.7)$ & $7.3(7.0,7.6)$ & $<0.001$ \\
\hline $2 \mathrm{~h}$ plasma glucose $(\mathrm{mmol} / \mathrm{l})$ & $5.5(4.6,6.3)$ & $6.3(5.4,7.1)$ & $8.4(8.2,9.2)$ & $8.9(8.4,9.8)$ & $10.6(7.6,12.3)$ & $<0.001$ \\
\hline Fasting serum insulin (pmol/l) & $31(21,43)$ & $42(30,63)$ & $35(29,51)$ & $51(36,71)$ & $58(43.0,86.0)$ & $<0.001$ \\
\hline $2 \mathrm{~h}$ serum insulin $(\mathrm{pmol} / \mathrm{l})$ & $135(83,209)$ & $186(110,283)$ & $304(189,485)$ & $380(253,490)$ & $366(190,582)$ & $<0.001$ \\
\hline $\mathrm{HbA}_{1 \mathrm{c}}(\mathrm{mmol} / \mathrm{mol})$ & $37.7(35.5,39.9)$ & $38.8(36.6,41.0)$ & $38.8(36.6,41.0)$ & $39.9(37.7,42.1)$ & $43.2(39.9,46.5)$ & $<0.001$ \\
\hline $\mathrm{HbA}_{1 \mathrm{c}}(\%)$ & $5.6(5.4,5.8)$ & $5.7(5.5,5.9)$ & $5.7(5.5,5.9)$ & $5.8(5.6,6.0)$ & $6.1(5.8,6.4)$ & $<0.001$ \\
\hline HOMA-IS (arbitrary unit) & $0.10(0.10,0.20)$ & $0.08(0.05,0.12)$ & $0.11(0.08,0.14)$ & $0.07(0.05,0.09)$ & $0.05(0.04,0.07)$ & $<0.001$ \\
\hline $\mathrm{ISI}_{0,120}$ (arbitrary unit) & $45.8(36.7,59.1)$ & $35.4(29.5,44.4)$ & $25.2(21.6,28.5)$ & $21.0(18.924 .4)$ & $16.7(13.4,27.4)$ & $<0.001$ \\
\hline BIGTT-AIR (arbitrary unit) & $1,454(1,163,1,827)$ & $1,092(897,1,454)$ & $1,475(1,217,1,826)$ & $1,248(1,040,1,626)$ & $1,031(745,1,390)$ & $<0.001$ \\
\hline DI (arbitrary unit $\left.\times 10^{3}\right)$ & $67.1(51.9,93.5)$ & $41.3(34.0,53.2)$ & $39.1(28.5,44.1)$ & $27.5(22.7,34.5)$ & $20.4(15.2,24.3)$ & $<0.001$ \\
\hline
\end{tabular}

Data are medians $\left(1^{\text {st }}\right.$ quartile- $3^{\text {rd }}$ quartile) or $n(\%)$

${ }^{a}$ Employed includes students and the self-employed. Out of work includes the unemployed, housewives, retired individuals and individuals receiving government provisions

Combined analysis of PAEE and CRF The interaction analyses showed that CRF did not modify the association between PAEE for any of the markers of glucose metabolism ( $p>0.05$ for all; data not shown). Table 3 shows the results from Model 4, in which PAEE and CRF were included in the same model (confounder adjustments similar to Model 3). Therefore, the interaction term was omitted from the model. When adjusting CRF for PAEE and vice versa, an association between both PAEE and CRF with fasting and $2 \mathrm{~h}$ serum insulin concentration and $\mathrm{ISI}_{0,120}$ remained significant.
Additionally, CRF was independently significantly associated with fasting and $2 \mathrm{~h}$ plasma glucose levels, HOMA-IS and DI.

\section{Discussion}

In a large population with objective measures of physical activity, we showed that neither CRF nor PAEE was different in individuals with $\mathrm{i}$-IFG as compared with those with NGT, but both were reduced in individuals with i-IGT, IFG + IGT
Fig. 1 Estimated daily PAEE (a), CRF (b) and METs $\geq 3.0$ (c) by glucose tolerance status. Data expressed as geometrical means $(95 \% \mathrm{CI})$

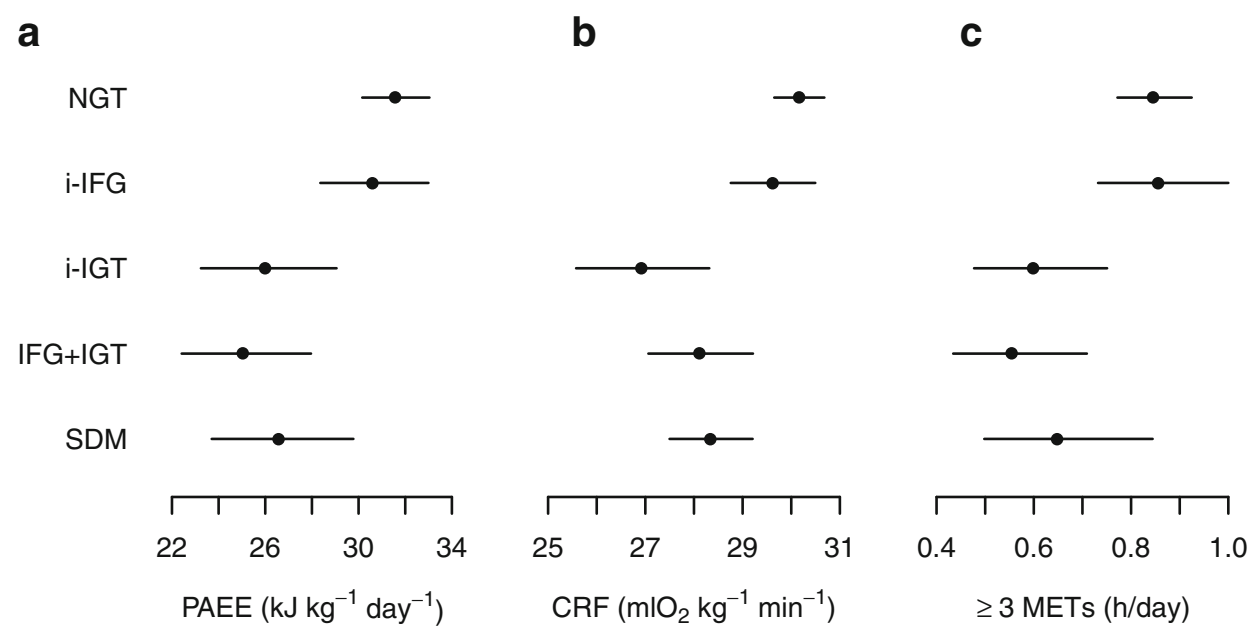


Table 2 Associations of PAEE vs CRF with markers of glucose metabolism

\begin{tabular}{|c|c|c|c|}
\hline Difference per standardised PAEE or CRF & Model 1 & Model 2 & Model 3 \\
\hline \multicolumn{4}{|c|}{ Difference per standardised PAEE ( $\beta$-estimates and $95 \% \mathrm{CI})$} \\
\hline Fasting plasma glucose $(\mathrm{mmol} / \mathrm{l})$ & $-0.09(-0.15,-0.03)^{* *}$ & $-0.08(-0.14,-0.02)^{* *}$ & $-0.02(-0.08,0.04)$ \\
\hline $2 \mathrm{~h}$ plasma glucose $(\mathrm{mmol} / \mathrm{l})$ & $-0.43(-0.60,-0.26)^{* * *}$ & $-0.42(-0.60,-0.25)^{* * *}$ & $-0.25(-0.42,-0.08)^{* *}$ \\
\hline Fasting serum insulin $(\mathrm{pmol} / 1)^{\mathrm{a}}$ & $-15(-18,-11)^{* * *}$ & $-15(-19,-11)^{* * *}$ & $-8(-12,-4)^{* * *}$ \\
\hline $2 \mathrm{~h}$ serum insulin $(\mathrm{pmol} / \mathrm{l})^{\mathrm{a}}$ & $-19(-24,-14)^{* * *}$ & $-19(-24,-14)^{* * *}$ & $-13(-18,-8)^{* * *}$ \\
\hline $\mathrm{HbA}_{1 \mathrm{c}}(\mathrm{mmol} / \mathrm{mol})$ & $-0.05(-0.40,0.31)$ & $-0.10(-0.46,0.27)$ & $0.21(-0.15,0.57)$ \\
\hline $\mathrm{HbA}_{1 \mathrm{c}}(\%)$ & $-0.00(-0.04,0.01)$ & $-0.01(-0.04,0.02)$ & $0.02(-0.01,0.05)$ \\
\hline HOMA-IS (\%) ${ }^{\mathrm{a}}$ & $18.8(13.0,24.9)^{* * *}$ & $18.9(13.0,25.3)^{* * *}$ & $9.0(4.1,14.2)^{* * *}$ \\
\hline $\operatorname{ISI}_{0,120}(\%)^{\mathrm{a}}$ & $13.9(9.9,18.1)^{* * *}$ & $13.6(9.5,17.9)^{* * *}$ & $8.0(4.4,11.9)^{* * *}$ \\
\hline BIGTT-AIR (\%) ${ }^{\mathrm{a}}$ & $-4.9(-7.9,-2.0)^{* *}$ & $-6.1(-9.0,-3.0)^{* * *}$ & $-2.7(-5.7,0.4)$ \\
\hline DI $(\%)^{\mathrm{a}}$ & $8.9(3.8,14.2)^{* * *}$ & $7.4(2.3,12.8)^{* *}$ & $5.7(0.5,11.1)^{* *}$ \\
\hline \multicolumn{4}{|c|}{ Difference per standardised CRF ( $\beta$-estimates and 95\% CI) } \\
\hline Fasting plasma glucose (mmol/l) & $-0.16(-0.22,-0.10)^{* * *}$ & $-0.17(-0.23,-0.10)^{* * *}$ & $-0.09(-0.16,-0.03)^{* *}$ \\
\hline $2 \mathrm{~h}$ plasma glucose $(\mathrm{mmol} / \mathrm{l})$ & $-0.55(-0.73,-0.37)^{* * *}$ & $-0.53(-0.71,-0.35)^{* * *}$ & $-0.31(-0.49,-0.13)^{* * *}$ \\
\hline Fasting serum insulin $(\mathrm{pmol} / 1)^{\mathrm{a}}$ & $-20(-24,-16)^{* * *}$ & $-20(-24,-16)^{* * *}$ & $-12(-16,-8)^{* * *}$ \\
\hline $2 \mathrm{~h}$ serum insulin $(\mathrm{pmol} / \mathrm{l})^{\mathrm{a}}$ & $-24(-29,-19)^{* * *}$ & $-24(-28,-19)^{* * *}$ & $-16(-21,-11)^{* * *}$ \\
\hline $\mathrm{HbA}_{1 \mathrm{c}}(\mathrm{mmol} / \mathrm{mol})$ & $-0.64(-1.01,-0.27)^{* * *}$ & $-0.60(-0.97,-0.22)^{* *}$ & $-0.23(-0.62,0.15)$ \\
\hline $\mathrm{HbA}_{1 \mathrm{c}}(\%)$ & $-0.06(-0.09,-0.03)^{* * *}$ & $-0.06(-0.09,-0.02)^{* *}$ & $-0.02(-0.06,0.01)$ \\
\hline HOMA-IS (\%) & $28.8(22.4,35.6)^{* * *}$ & $28.4(21.8,35.3)^{* * *}$ & $15.0(9.6,20.7)^{* * *}$ \\
\hline $\operatorname{ISI}_{0,120}(\%)^{\mathrm{a}}$ & $18.6(14.3,23.1)^{* * *}$ & $18.2(13.8,22.70)^{* * *}$ & $10.9(6.9,15.0)^{* * *}$ \\
\hline BIGTT-AIR (\%) $)^{\mathrm{a}}$ & $-4.6(-7.8,-1.5)^{* *}$ & $-4.6(-7.8,-1.4)^{* *}$ & $0.1(-3.2,3.4)$ \\
\hline DI $(\%)^{\mathrm{a}}$ & $13.3(7.9,19.0)^{* * *}$ & $12.8(7.4,18.7)^{* * *}$ & $11.1(5.4,17.1)^{* * *}$ \\
\hline
\end{tabular}

The standard deviation of PAEE was $15.7 \mathrm{~kJ} \mathrm{~kg}^{-1}$ day ${ }^{-1}$. The standard deviation of CRF was $5.3 \mathrm{ml} \mathrm{O}_{2} \mathrm{~kg}^{-1} \mathrm{~min}^{-1}$. Model 1: adjusted for age and sex; Model 2: adjusted for age, sex, smoking status and alcohol intake; Model 3: adjusted for age, sex, smoking, alcohol intake and waist circumference

${ }^{a}$ Estimates from serum insulin and derived indices were back-transformed from naturally log-transformed values and are presented as relative changes

$* * p<0.01$

$* * * p<0.001$

Table 3 Combined analysis of PAEE and CRF with markers of glucose metabolism

\begin{tabular}{|c|c|c|}
\hline $\begin{array}{l}\text { Difference per standardised PAEE } \\
\text { and CRF ( } \beta \text {-estimates and } 95 \% \mathrm{CI})\end{array}$ & $\begin{array}{l}\text { PAEE } \\
\left(\mathrm{kJ} \mathrm{kg}^{-1} \text { day }^{-1}\right)\end{array}$ & $\begin{array}{l}\mathrm{CRF} \\
\left(\mathrm{ml} \mathrm{O}_{2} \mathrm{~kg}^{-1} \mathrm{~min}^{-1}\right)\end{array}$ \\
\hline Fasting plasma glucose $(\mathrm{mmol} / \mathrm{l})$ & $0.01(-0.05,0.07)$ & $-0.10(-0.16,-0.03)^{* *}$ \\
\hline $2 \mathrm{~h}$ plasma glucose $(\mathrm{mmol} / \mathrm{l})$ & $-0.17(-0.35,0.02)$ & $-0.25(-0.44,-0.05)^{*}$ \\
\hline Fasting serum insulin $(\mathrm{pmol} / \mathrm{l})^{\mathrm{a}}$ & $-5(-9,-0.3)^{*}$ & $-10(-14,-8)^{* * *}$ \\
\hline $2 \mathrm{~h}$ serum insulin $(\mathrm{pmol} / \mathrm{l})^{\mathrm{a}}$ & $-9(-14,-3)^{* *}$ & $-14(-19,-8)^{* * *}$ \\
\hline $\mathrm{HbA}_{1 \mathrm{c}}(\mathrm{mmol} / \mathrm{mol})$ & $0.33(-0.06,0.71)$ & $-0.35(-0.76,0.06)$ \\
\hline $\mathrm{HbA}_{1 \mathrm{c}}(\%)$ & $0.03(-0.01,0.07)$ & $-0.03(-0.07,0.01)$ \\
\hline HOMA-IS (\%) ${ }^{a}$ & $4.7(-0.2,9.9)$ & $13.1(7.2,19.1)^{* * *}$ \\
\hline $\operatorname{ISI}_{0,120}(\%)^{\mathrm{a}}$ & $5.1(1.3,9.0)^{* *}$ & $8.9(4.7,13.2)^{* * *}$ \\
\hline BIGTT-AIR $(\%)^{\mathrm{a}}$ & $-3.1(-6.3,0.2)$ & $1.2(-2.3,4.9)$ \\
\hline DI $(\%)^{\mathrm{a}}$ & $2.3(-3.0,7.9)$ & $10.2(4.2,16.5)^{* * *}$ \\
\hline
\end{tabular}

Model 4: adjusted for age, sex, smoking, alcohol intake, waist circumference, PAEE and fitness

${ }^{a}$ Estimates from serum insulin and derived indices were back-transformed from naturally log-transformed values and are presented as relative changes

$* p<0.05$

$* * p<0.01$

$* * * p<0.001$ 
and SDM. PAEE was inversely associated with $2 \mathrm{~h}$ plasma glucose, fasting and $2 \mathrm{~h}$ serum insulin levels, insulin sensitivity index and DI after adjustment for potential confounders. CRF was associated with fasting and $2 \mathrm{~h}$ plasma glucose and serum insulin concentrations, insulin sensitivity and DI. We found no evidence of a modifying effect of CRF on the relationship between PAEE and markers of glucose metabolism. When adjusting PAEE for CRF and vice versa, both exposures were associated with insulin levels and the insulin sensitivity index, but only CRF was independently associated with lower fasting and $2 \mathrm{~h}$ glucose levels, higher HOMA-IS and improved beta cell function.

The finding of similar levels of PAEE and CRF in individuals with i-IFG and NGT, but lower levels in the i-IGT group, may explain the different pathophysiology of individuals with i-IFG vs i-IGT observed in previous studies [4, 6-8]. The lower PAEE and the lower amount of time spent in activities of moderate-to-vigorous intensity ( $\geq 3$ METs) observed in the i-IGT group was partly explained by a higher proportion of women, higher age and waist circumference, which supports previous findings [30]. Individuals with i-IGT, IFG + IGT and SDM only spent $2.6 \%$ of the time in activities of moderate-to-vigorous intensity ( $\geq 3$ METs), which could partly explain the lower CRF levels. It has been suggested that the improvement in glucose metabolism in response to exercise training is not uniform across the subgroups of individuals with prediabetes or type 2 diabetes. For instance, individuals with IFG + IGT do not experience the same beneficial effects of exercise on $2 \mathrm{~h}$ glucose levels and insulin sensitivity as individuals with i-IGT [9]. In another study, approximately $15-20 \%$ of individuals with type 2 diabetes failed to improve their glucose homeostasis and insulin sensitivity with exercise training [31]. Large-scale clinical intervention studies including habitual monitoring of behaviour are needed to advance our ability to understand and identify responders vs nonresponders to exercise in order to optimise prevention and treatment strategies for type 2 diabetes.

CRF, but not PAEE, was associated with a small but significant difference in fasting plasma glucose levels in the fully adjusted model. This finding was unexpected because of the similar CRF levels in individuals with i-IFG and NGT in our study population. A previous study showed no association of CRF with fasting glucose concentrations in prediabetic men and women [32]. However, in a prospective study, CRF was associated with progression from NGT to i-IFG, suggesting that CRF may play a role in the regulation of fasting glucose concentration over time [33]. The association of CRF with $2 \mathrm{~h}$ plasma glucose concentration confirms previous observations $[32,34]$. Many studies have also documented relationships between PAEE and $2 \mathrm{~h}$ plasma glucose levels independent of the level of CRF $[17,35,36]$. In a previous analysis of participants from the ADDITION-PRO cohort, the association between PAEE and $2 \mathrm{~h}$ plasma glucose was only borderline significant when potential confounders were taken into account [22]. A possible reason for the slightly different findings in this subpopulation of ADDITION-PRO and the previous results is that adjustment for baseline diabetes risk was not performed in the current analysis because it was considered to be a precursor rather than a confounder for the association between CRF and glucose metabolism.

In this study, PAEE and CRF were both positively associated with $\mathrm{ISI}_{0,120}$ independent of each other, indicating additive effects. ISI $_{0,120}$ includes $2 \mathrm{~h}$ post-OGTT plasma glucose and serum insulin concentrations in the calculation, and it mainly reflects peripheral or whole-body insulin sensitivity [37]. By contrast, the HOMA-IS model is based on fasting plasma glucose and serum insulin concentrations and therefore primarily reflects hepatic insulin sensitivity [37]. The findings of associations of PAEE and CRF with peripheral insulin sensitivity are consistent with previous findings using objectively measured PAEE [38, 39], including previous analyses on participants in the ADDITION-PRO cohort [22]. A recent randomised controlled trial showed that interval walking training improved insulin sensitivity more than continuous walking training, which further supports our finding of an association between $\mathrm{CRF}$ and insulin sensitivity independent of PAEE [40]. With regard to HOMA-IS, adjustment for CRF made the association between PAEE and HOMA-IS nonsignificant. Together, these observations suggest that part of the effect of PAEE on hepatic insulin sensitivity is mediated through CRF. However, more studies using detailed physiological measures are needed to clarify the relative roles of PAEE vs CRF on glucose metabolism in the liver.

Our finding of a significant association between CRF and DI is in agreement with that of a previous cross-sectional study [41]. However, the effect of CRF on DI seems to be dependent on the remaining beta cell capacity. The dose-response relationship between CRF and improvement of beta cell function appears to be linear in individuals with prediabetes [42], whereas a higher effect of CRF on beta cell function has been found in diabetic individuals with moderate insulin secretion than in those with poor insulin secretion [43, 44].

A significant association between $\mathrm{CRF}$ and $\mathrm{HbA}_{1 \mathrm{c}}$ was found, but adjustment for waist circumference attenuated the association. For PAEE, there was no significant association with $\mathrm{HbA}_{1 \mathrm{c}}$, but the association switched direction when adjusting for waist circumference. These findings indicate that waist circumference strongly confounds the relationships of both CRF and PAEE with $\mathrm{HbA}_{1 \mathrm{c}}$. In the randomised controlled trial Look AHEAD [45], a lifestyle intervention focused on weight loss through decreased caloric intake and increased physical activity was associated with reductions in waist circumference and improvements in $\mathrm{CRF}$ and $\mathrm{HbA}_{1 \mathrm{c}}$ during the first year of follow-up [45]. This result suggests that the improvement in $\mathrm{HbA}_{1 \mathrm{c}}$ was partly mediated through reductions in waist circumference. 
A major strength of our study is that PAEE was estimated using combined accelerometry and heart rate monitoring. This method made it possible to estimate activity intensity or instantaneous energy expenditure above rest, which when integrated over time yields average daily PAEE or total volume of activity. Both the activity volume and intensity estimate have been shown to be more valid than estimates based on either heart rate or accelerometry alone, in both experimental and free-living conditions as compared with indirect calorimetry and doubly-labelled water, particularly when heart rate is individually calibrated [23, 24, 46-48]. However, both PAEE and CRF reflect multidimensional activities with an inherent variability, which makes their estimation difficult, particularly for PAEE [34]. CRF reflects a longterm dimension of the physical activity level of the participants as well as a genetic component [34]. In this study, there was a trend towards selection of a healthier subset of the ADDITION-PRO participants. This selection might limit the generalisability of the results to other populations with a similar 'westernised' adult population and at varying risk of diabetes. However, it is unlikely that this selection would have introduced bias to the observed associations.

In conclusion, we found that individuals with i-IFG had normal PAEE and CRF levels. By contrast, individuals with i-IGT, IFG + IGT or SDM had lower PAEE and CRF levels than those with NGT. Both PAEE and CRF were associated with improved insulin sensitivity independent of each other. CRF did not modify the association of PAEE with any markers of glucose metabolism. CRF, but not PAEE, was associated with lower plasma glucose levels and improved beta cell function, suggesting that the effect of PAEE on glucose metabolism is mediated through improvements in CRF. These findings indicate that long-term accumulation of PAEE and/or the intensity of physical activity are particularly important for glucose regulation. Future prospective studies are needed to investigate the association between changes in PAEE, sedentary behaviour and time spent in vigorous intensity activity in relation to changes in glucose metabolism.

Acknowledgements We acknowledge K. Borch-Johnsen (Chief Medical Officer, Holbæk Hospital, Denmark) and A. Sandbæk (Department of Public Health - Institute of General Medical Practice, Aarhus University, Denmark) for their contribution to the design of the ADDITIONDenmark and ADDITION-PRO studies. We thank the ADDITIONDenmark study centres, general practitioners and participants for their contribution to the study. D. Vistisen (Steno Diabetes Center) is thanked for her support with statistical analysis.

Funding The ADDITION-Denmark study was supported by the National Health Services in the counties of Copenhagen, Aarhus, Ringkøbing, Ribe and Southern Jutland in Denmark; the Danish Council for Strategic Research; the Danish Research Foundation for General Practice; Novo Nordisk Foundation; the Danish Centre for Evaluation and Health Technology Assessment; the Diabetes Fund of the National Board of Health; the Danish Medical Research Council; and the Aarhus University Research Foundation. Additionally, the
ADDITION-PRO study was funded by an unrestricted grant from the European Foundation for the Study of Diabetes/Pfizer for Research into Cardiovascular Disease Risk Reduction in Patients with Diabetes (74550801), the Danish Council for Strategic Research, and internal research and equipment funds from Steno Diabetes Center. SB and DLC were funded by the UK Medical Research Council (grant MC_UU_12015/3). NBJ and DRW are funded by the Danish Diabetes Academy, supported by the Novo Nordisk Foundation.

Duality of interest LPL, KF, NBJ and MEJ are employed by Steno Diabetes Center A/S, a research hospital working in the Danish National Health Service and owned by Novo Nordisk A/S. Steno Diabetes Center receives part of its core funding from unrestricted grants from the Novo Foundation and Novo Nordisk A/S. KF, NBJ, DRW, MEJ and ALSH own shares in Novo Nordisk A/S. No other potential conflicts of interest relevant to this article were reported. The other authors declare that there is no duality of interest associated with their contribution to this manuscript.

Contribution statement LPL, KF and DLC conceived the idea, researched and interpreted data, and drafted the manuscript. MEJ contributed to analysis and interpretation of data. ALSH and SB processed accelerometer and heart rate data. NBJ, DRW and TL designed the ADDITION-PRO study, contributed to discussion and reviewed and edited the manuscript. MEJ, SB and ALSH contributed to discussion and reviewed and edited the manuscript. All authors approved the final version of the manuscript. LPL is the guarantor of this work and, as such, had full access to all the data in the study and takes responsibility for the integrity of the data and the accuracy of the data analysis.

\section{References}

1. Virally M, Blicklé J-F, Girard J et al (2007) Type 2 diabetes mellitus: epidemiology, pathophysiology, unmet needs and therapeutical perspectives. Diabetes Metab 33:231-244

2. Stumvoll M, Goldstein BJ, van Haeften TW (2005) Type 2 diabetes: principles of pathogenesis and therapy. Lancet 365:1333-1346

3. Færch K, Witte DR, Tabák AG et al (2013) Trajectories of cardiometabolic risk factors before diagnosis of three subtypes of type 2 diabetes: a post-hoc analysis of the longitudinal Whitehall II cohort study. Lancet Diabetes Endocrinol 1:43-51

4. Færch K, Johansen NB, Witte DR et al (2014) Relationship between insulin resistance and beta cell dysfunction in subphenotypes of pre-diabetes and type 2 diabetes. J Clin Endocrinol Metab 100: 707-716

5. Tabák AG, Herder C, Rathmann W et al (2012) Prediabetes: a highrisk state for diabetes development. Lancet 379:2279-2290

6. Færch K, Borch-Johnsen K, Holst JJ, Vaag A (2009) Pathophysiology and aetiology of impaired fasting glycaemia and impaired glucose tolerance: does it matter for prevention and treatment of type 2 diabetes? Diabetologia 52:1714-1723

7. Færch K, Vaag A, Holst JJ et al (2009) Natural history of insulin sensitivity and insulin secretion in the progression from normal glucose tolerance to impaired fasting glycemia and impaired glucose tolerance: the Inter99 study. Diabetes Care 32:439-444

8. Abdul-Ghani MA, Jenkinson CP, Richardson DK et al (2006) Insulin secretion and action in subjects with impaired fasting glucose and impaired glucose tolerance: results from the Veterans Administration Genetic Epidemiology Study. Diabetes 55:1430 1435

9. Malin SK, Kirwan JP (2012) Fasting hyperglycaemia blunts the reversal of impaired glucose tolerance after exercise training in obese older adults. Diabetes Obes Metab 14:835-841 
10. Paffenbarger RS, Blair SN, Lee IM, Hyde RT (1993) Measurement of physical activity to assess health effects in free-living populations. Med Sci Sports Exerc 25:60-70

11. Caspersen CJ, Powell KE, Christenson GM (1985) Physical activity, exercise, and physical fitness: definitions and distinctions for health-related research. Public Health Rep 100:126-131

12. Hu G, Lakka TA, Kilpeläinen TO, Tuomilehto J (2007) Epidemiological studies of exercise in diabetes prevention. Appl Physiol Nutr Metab 32:583-595

13. Gill JMR (2007) Physical activity, cardiorespiratory fitness and insulin resistance: a short update. Curr Opin Lipidol 18:47-52

14. Lee IM, Paffenbarger RS Jr (2000) Associations of light, moderate, and vigorous intensity physical activity with longevity. The Harvard Alumni Health Study. Am J Epidemiol 151:293-299

15. Houmard JA, Tanner CJ, Slentz CA et al (2004) Effect of the volume and intensity of exercise training on insulin sensitivity. J Appl Physiol (1985) 96:101-106

16. Karstoft K, Winding K, Knudsen SH et al (2013) The effects of free-living interval-walking training on glycemic control, body composition, and physical fitness in type 2 diabetic patients: a randomized, controlled trial. Diabetes Care 36:228-236

17. Assah FK, Ekelund U, Brage S et al (2009) Free-living physical activity energy expenditure is strongly related to glucose intolerance in Cameroonian adults independently of obesity. Diabetes Care 32: 367-369

18. Jiménez-Pavón D, Ruiz JR, Ortega FB et al (2013) Physical activity and markers of insulin resistance in adolescents: role of cardiorespiratory fitness levels - the HELENA study. Pediatr Diabetes 14: 249-258

19. O'Donovan G, Hillsdon M, Ukoumunne OC et al (2013) Objectively measured physical activity, cardiorespiratory fitness and cardiometabolic risk factors in the Health Survey for England. Prev Med 57:201-205

20. Franks PW, Ekelund U, Brage S et al (2004) Does the association of habitual physical activity with the metabolic syndrome differ by level of cardiorespiratory fitness? Diabetes Care 27:1187-1193

21. Johansen NB, Hansen A-LS, Jensen TM et al (2012) Protocol for ADDITION-PRO: a longitudinal cohort study of the cardiovascular experience of individuals at high risk for diabetes recruited from Danish primary care. BMC Public Health 12:1078

22. Hansen A-LS, Carstensen B, Helge JW et al (2013) Combined heart rate- and accelerometer-assessed physical activity energy expenditure and associations with glucose homeostasis markers in a population at high risk of developing diabetes: the ADDITION-PRO study. Diabetes Care 36:3062-3069

23. Brage S, Brage N, Franks PW et al (2004) Branched equation modeling of simultaneous accelerometry and heart rate monitoring improves estimate of directly measured physical activity energy expenditure. J Appl Physiol (1985) 96:343-351

24. Brage S, Ekelund U, Brage $\mathrm{N}$ et al (2007) Hierarchy of individual calibration levels for heart rate and accelerometry to measure physical activity. J Appl Physiol (1985) 103:682-692

25. McArdle WD (1996) Exercise physiology: energy, nutrition, and human performance, 4th edn. Williams \& Wilkins, Baltimore

26. Tanaka H, Monahan KD, Seals DR (2001) Age-predicted maximal heart rate revisited. J Am Coll Cardiol 37:153-156

27. WHO (2006) Definition and diagnosis of diabetes mellitus and intermediate hyperglycemia. http://whqlibdoc.who.int/ publications/2006/9241594934 eng.pdf. Accessed 15 Oct 2014

28. Gutt M, Davis CL, Spitzer SB et al (2000) Validation of the insulin sensitivity index $(\operatorname{ISI}(0,120))$ : comparison with other measures. Diabetes Res Clin Pract 47:177-184

29. Hansen T, Drivsholm T, Urhammer SA et al (2007) The BIGTT test: a novel test for simultaneous measurement of pancreatic beta-cell function, insulin sensitivity, and glucose tolerance. Diabetes Care 30:257-262

30. Ferraro R, Lillioja S, Fontvieille AM et al (1992) Lower sedentary metabolic rate in women compared with men. J Clin Invest 90:780 784

31. Stephens NA, Sparks LM (2015) Resistance to the beneficial effects of exercise in type 2 diabetes: are some individuals programmed to fail? J Clin Endocrinol Metab 100:43-52

32. Gatterer H, Ulmer H, Dzien A et al (2011) High cardiorespiratory fitness is more beneficial in pre-diabetic men than women. Clinics 66:747-751

33. Wei M, Gibbons LW, Mitchell TL et al (1999) The association between cardiorespiratory fitness and impaired fasting glucose and type 2 diabetes mellitus in men. Ann Intern Med 130:89-96

34. Wareham NJ, Wong MY, Day NE (2000) Glucose intolerance and physical inactivity: the relative importance of low habitual energy expenditure and cardiorespiratory fitness. Am J Epidemiol 152: 132-139

35. Healy GN, Dunstan DW, Shaw JE et al (2006) Beneficial associations of physical activity with 2-h but not fasting blood glucose in Australian adults: the AusDiab study. Diabetes Care 29:2598-2604

36. Kriska AM, LaPorte RE, Pettitt DJ et al (1993) The association of physical activity with obesity, fat distribution and glucose intolerance in Pima Indians. Diabetologia 36:863-869

37. Abdul-Ghani MA, Matsuda M, Balas B, DeFronzo RA (2007) Muscle and liver insulin resistance indexes derived from the oral glucose tolerance test. Diabetes Care 30:89-94

38. Balkau B, Mhamdi L, Oppert J-M et al (2008) Physical activity and insulin sensitivity: the RISC study. Diabetes 57:2613-2618

39. Larsson CA, Krøll L, Bennet L et al (2012) Leisure time and occupational physical activity in relation to obesity and insulin resistance: a population-based study from the Skaraborg Project in Sweden. Metabolism 61:590-598

40. Karstoft K, Winding K, Knudsen SH et al (2014) Mechanisms behind the superior effects of interval vs continuous training on glycaemic control in individuals with type 2 diabetes: a randomised controlled trial. Diabetologia 57:2081-2093

41. Solomon T, Malin S, Knudsen S et al (2015) Association between cardiorespiratory fitness and the determinants of glycemic control across the entire glucose tolerance continuum. Diabetes Care 38: 921-929

42. Malin SK, Solomon TPJ, Blaszczak A et al (2013) Pancreatic $\beta$-cell function increases in a linear dose-response manner following exercise training in adults with prediabetes. Am J Physiol Endocrinol Metab 305:E1248-E1254

43. Dela F, von Linstow ME, Mikines KJ, Galbo H (2004) Physical training may enhance beta-cell function in type 2 diabetes. Am J Physiol Endocrinol Metab 287:E1024-E1031

44. Slentz CA, Tanner CJ, Bateman LA et al (2009) Effects of exercise training intensity on pancreatic beta-cell function. Diabetes Care 32:1807-1811

45. Wing RR, Bolin P, Brancati FL et al (2013) Cardiovascular effects of intensive lifestyle intervention in type 2 diabetes. N Engl J Med 369:145-154

46. Brage S, Brage N, Franks PW et al (2005) Reliability and validity of the combined heart rate and movement sensor Actiheart. Eur J Clin Nutr 59:561-570

47. Villars C, Bergouignan A, Dugas J et al (2012) Validity of combining heart rate and uniaxial acceleration to measure free-living physical activity energy expenditure in young men. J Appl Physiol (1985) 113:1763-1771

48. Crouter SE, Churilla JR, Bassett DR Jr (2008) Accuracy of the Actiheart for the assessment of energy expenditure in adults. Eur J Clin Nutr 62:704-711 